\section{Gérard Fayolle \\ Les nouvelles ruralité : les villages et la mondialisation Luçon, Éditions du Sud-Ouest, 2001, 251 p.}

On l'aura deviné, le Sud-ouest en question n'a rien à voir avec la région limitrophe de notre chère capitale fédérale. En fait, cet ouvrage a été imprimé à Luçon, en Vendée, à mi-chemin entre Le Puy-du-Fou et les Sables-d'Olonne. Une région que j'aime bien, entre autres pour les noms de lieu que l'on ne trouve nulle part ailleurs. Découvert sur la toile, j'ai pensé que la lecture de cet ouvrage s'imposait étant donné la mise en œuvre au Québec, depuis 2002, de ce que l'on ose encore appeler la Politique nationale de la ruralité. D'ailleurs, dès le début, on y trouve une allusion à notre Belle Province où la civilisation rurale en voie de disparition n'empêche pas les élites de Montréal et les hommes politiques de Québec de reconnaître ce qu'ils lui doivent : leur survie en tant qu'entité culturelle. L'auteur souligne que les Québécois ont été les premiers à devoir faire face à la brillante et redoutable civilisation anglo-saxonne. Et comment! On n'a qu'à voir nos étudiants se défendre dans la langue de Shakespeare... Doit-on s'en réjouir ?

Originaire de la Dordogne rurale, l'auteur est un élu depuis des lustres, président du Conseil général, sénateur et quoi encore ? Plus intéressant pour nous sont ses quelque dix ouvrages à caractère historique portant pour la plupart sur le Périgord de mon ancêtre (le truchement Jacques Joyal). En considérant que les valeurs propres à la société rurale sont menacées, l'auteur se donne pour objectif de montrer comment, en s'organisant en conséquence, le village peut en assurer la survie. En se voulant un plaidoyer pour les libertés locales et celles des régions, l'intérêt de l'ouvrage, pour les cousins que nous sommes, apparait évident.

Bien sûr, il faut d'abord tenter de définir ce qu'est le rural. Et l'incontournable anecdote, hélas trop sérieuse, qui veut qu'est rural ce qui n'est pas urbain, se retrouve ici. Et toujours en relation avec notre vécu, plusieurs allusions à la place qu'occupent les néoruraux offrent une historique qui remonte à la fin des année 1960 caractérisées par l'essor de la mise en œuvre du «modèle fromage de chèvre ». Oui, ce qu'ils la trouvaient belle cette Gaspésie ces hippies de Montréal inspirés par les événement de mai 68. Mais, comme l'écrit Gérard Fayolle, après les premiers enthousiasmes arrivent les premiers échecs, car la vie des bergers des Cévennes (ou de la vallée de la Matapédia) est dure, surtout en hiver, et davantage pour des amateurs arrivés sans moyens du $6^{\mathrm{e}}$ arrondissement (ou du Faubourg à M'lasse). Il faudra donc compter sur d'autres acteurs pour défendre la ruralité. Et l'auteur les identifie dans un chapitre intitulé Les avocats de la ruralité.

Parmi ces derniers, Jean Giono occupe une place de choix. Inutile d'insister, c'est pourquoi je retiens plutôt un certain Joseph Delteil qui, il y a quarante ans, en décrivant la cuisine paysanne, évoquait les cochonnailles de janvier, les truffes de février, les bigarreaux et les dindonneaux de juin, les cailles de juillet, les écrevisses d'août, les figues et les cèpes de septembre le chapon à l'ail de novembre et les huîtres de Marennes en décembre pour le Réveillon. Mais quoi !, dira-t-on, nous avons bien les petits poissons des chenaux en janvier, le homard des Îles en juin, le blé d'Inde deux couleurs en août, les huîtres de Carraquet en septembre, la tire d'éponge et Sainte Catherine en novembre, et que dire du gâteau aux fruits de décembre ! Oui, à chacun sa ruralité !

C'est cependant la troisième partie : La liberté des villages, qui touche le plus le lecteur québécois. Si le clocher en demeure le symbole, c'est son maire qui constitue le cœur du système. On n'a qu'à penser aux défis que rencontrent quotidiennement les maires de nos villages de moins de 1000 habitants. Tant que le maire existe, la ruralité demeure, écrit Gérard Fayolle qui, avec ô combien de pertinence, lui rend un vibrant hommage. Vient ensuite le concept de «pays » dont l'auteur semble ignorer qu'il s'agit d'un héritage gallo-romain (pagii). C'est plus ou moins l'équivalent de nos MRC, avec parfois des noms aussi évocateurs. C'est le lieu où élus et acteurs du développement se rencontrent pour, dans la mesure du possible, prendre en main une partie de leur destinée. Mais gare aux enchevêtrements de responsabilités, est-il signalé. On en connaît quelque chose au Québec avec nos multiples divisions régionales et compétences diverses.

Enfin, la section Ruralité et régions s'avère d'un grand intérêt. Aux yeux de l'auteur, la région doit affirmer son identité, démontrer sa légitimité et retrouver ses racines. Son avenir et celui de la ruralité se trouvent étroitement reliés. L'un ne va pas sans l'au- 
tre. En évoquant la nécessaire décentralisation des pouvoirs, l'auteur écrit que la ruralité n'a que des amis qui lui veulent du bien. Ouf! On sait parfois qu'avec certains amis on peut se priver d'ennemis. Passons. En se positionnant parmi les amis de la ruralité, le lecteur amoureux de celle-ci et de la France profonde ne pourra que se délecter à la lecture de cet ouvrage passionnant.

\section{Bernard Jouve, sous la direction de, Les politiques de déplacements urbains en Europe : l'innovation en question dans cinq villes européennes, Paris, L'Harmattan, 2003, 190 p.}

En quoi le transport urbain en Europe peut-il nous concerner? Pas évident à première vue, mais quand on prend en considération la situation, pas toujours facile, qui prévaut à Montréal et dans la plupart de nos villes moyennes, l'expérience vécue sous d'autres cieux ne peut qu'être d'une certaine utilité, pour ne pas dire d'une utilité certaine. On n'a qu'à imaginer ce cycliste empruntant une des nombreuses pistes, dont Trois-Rivières s'enorgueillit, et qui regarde de façon sceptique passer près de lui un gigantesque autobus de la société de transport en commun complètement vide. Est-ce que ça a du sens, se demandet-il en s'interrogeant sur ce qui se fait ailleurs? Sans en être vraiment conscient, Bernard Jouve, professeur au département de géographie de l'UQAM et titulaire de la Chaire de recherche du Canada en étude des dynamiques territoriales, en prenant la responsabilité de cet ouvrage, avait pour ambition de fournir des réponses à tout cycliste urbain en quête de sens. Pour ce faire, on offre au lecteur l'exemple de Genève, Lyon, Munich, Naples et Suttgart avec, à chaque fois, la contribution d'un spécialiste familier avec chacun de ces exemples.

En signalant que les pouvoirs publics ont surtout investi massivement dans les réseaux lourds de transport collectif (métro, tramway, trains régionaux), Jouve se demande si, en évoquant les politiques de déplacements urbains, en lieu et place de politiques de transports urbains, on sous-entend que l'on pense agir différemment à la fois sur le cadre et sur le contenu des politiques publiques. Est-ce le cas dans les différentes villes étudiées ? C'est la question centrale à laquelle l'ouvrage cherche à répondre. En fait, le lecteur se voit ici offrir le fondement des politiques en matière de déplacements urbains observées dans les cinq villes retenues comme autant d'études de cas.

Un élément qui retient l'attention avec tout ce qui s'écrit et se dit sous d'autres cieux en se rapportant aux fameux budgets participatifs de Porto Alegre, c'est l'importance qu'accorde Jouve à la démocratie participative. Il signale, en effet, que dans la majorité des villes ici étudiées, les politiques mises en place furent accompagnées d'une réflexion approfondie sur la démocratie locale et le rôle qui revient à cette chère société civile, pour parler comme Gramsci ou ses disciples québécois. Or, quand on évoque la participation populaire, on le devine bien, les considérations environnementales ont de meilleures chances d'être prises en compte. Il en est donc question ici et là à travers l'ouvrage. Et c'est ici que l'image du cycliste de Trois-Rivières ou d'ailleurs trouve sa pertinence, comme en témoigne l'exemple de Munich.

Cette ville, qui s'est vu attribuer le prix européen de la mobilité en 2000, s'est distinguée entre autres choses par l'opération "Appelez un vélo» ou «Call-abike ». Il s'agissait, à la faveur d'une promotion très bien conduite, de louer 2000 vélos de couleurs voyantes (ce qui change des sinistres vélos noirs d'Amsterdam) comme moyen de transport public individuel. L'histoire ne dit pas si le casque était fourni. Hélas, malgré un intérêt très rapidement manifesté par les citoyens (ou à cause de cela), l'opération s'est avérée à la fois trop complexe à gérer et non rentable. Car il est vite apparu que, pour joindre les deux bouts, il aurait fallu recourir à la contribution des villes limitrophes (tiens donc !), à défaut de quoi les Munichois se seraient vus forcés de payer pour des services dont bénéficieraient en grande partie des résidants desdites villes (retiens donc !). Doit-on y voir un argument en faveur des fusions municipales ? L'auteur, ignorant de la réalité québécoise, n'a pu faire un rapprochement avec une situation qui nous est bien familière. En fait, l'auteur termine sa contribution en signalant que, malgré la très forte mobilisation qu'elle a générée, la nouvelle politique des déplacements urbains à Munich souffre de l'absence d'un leadership fort de nature à stabiliser et à pérenniser le nouveau mode de régulation politique. Encore une fois, le lecteur québécois peut faire les rapprochements qu'il juge pertinents. L'ouvrage lui fournit d'ailleurs beaucoup d'occasions de le faire. 